\title{
Review of Single-Phase Grounding Fault Locating in Distribution Network
}

\author{
Bangtong $\mathrm{Li}^{1}$, Yanqi Zhang ${ }^{2}$, Rirui Huang ${ }^{1}$, Tao $\mathrm{Lin}^{2}$, Ning $\mathrm{Su}^{1}$, Rusi Chen ${ }^{2}$ \\ ${ }^{I}$ Qionghai power supply bureau, Hainan power grid company, Qionghai China \\ ${ }^{2}$ School of Electrical Engineering, Wuhan University, Wuhan China \\ *Corresponding Author: Tao Lin, School of Electrical Engineering, Wuhan University, Wuhan China
}

\begin{abstract}
The probability of single-phase grounding fault is the highest in the distribution network, however the locating of single-phase grounding fault in distribution network has not yet been satisfactorily resolved. Therefore, it is of great significance to study the fault locating of single-phase grounding in the distribution network. So far, a variety of locating methods are proposed and the relevant devices are developed. This paper reviews mainstream locating technologies, introduces the principle, the advantages and the disadvantages of each method briefly.
\end{abstract}

Keywords: Fault locating; Distribution network; Single-phase grounding fault; locating method

\section{INTRODUCTION}

Power system consists of four parts: generation, transmission, distribution and user. At present, the transmission network has been developed into multi-power ring network structure and its reliability is high. However, the distribution network adopts closed-loop structure and open-loop operation mode, so its reliability is lower. Once the distribution network fails, the power outage will occur. Nowadays, more and more attention has been paid to reliability of power supply, so it is quite necessary to research reliability of distribution network ${ }^{[1]}$.

In non-effectively earthed distribution network, the line fault is mainly divided into face- face fault and single-phase grounding fault. Because of the large short circuit current and the obvious fault path, so if equipped with the testing equipment and communication facilities, the face-face fault will be easy to locate and quickly isolated. However, due to complex structure of distribution network and minor fault current, the locating of single-phase grounding fault has not yet been satisfactorily resolved. In addition, the statistics show that the probability of single-phase grounding fault is the highest in the distribution network ${ }^{[2]}$. Therefore, it is of great significance to study the fault locating of single-phase grounding in the distribution network.

\section{Single-Phase Grounding Fault Locating Status}

About the study of single-phase grounding fault locating, the former Soviet Union putted forward amplitude comparison method based on overflows and reactive power direction. The French putted forward the grounding fault protection based on zero sequence admittance. And the Japan chosen the method based on reactive power direction. Besides, some foreign countries have applied the principle of artificial neural network to locate, and applied expert system methods according some references. A variety of locating methods are proposed and relevant devices are developed. So far, according to the different signals that analyzed, they can be divided into signal injection method, fault locating method based on steady state signals and fault locating method based on transient signals ${ }^{[3]}$. Further, the signal injection method can be divided into large signal injection method and small signal injection method; the fault locating method based on steady state signals can be divided into zero-sequence current comparison of amplitude ${ }^{[4]}$, zero-sequence current comparison of phase, method based on active power component of zero-sequence current ${ }^{[5]}$ and method based on negative-sequence current ${ }^{[6]}$; the fault locating method based on transient signals can be divided into the first half wave $\operatorname{method}^{[7]}$, the method based on transient feature frequency band ${ }^{[8]}$ and the method based on wavelet analysis ${ }^{[9]}$. The following review of single-phase grounding fault locating will be presented, and each method will be introduced briefly.

International Journal of Research Studies in Electrical and Electronics Engineering (IJRSEEE) Page | 1 


\section{Introduction OF Single-PhaSe Grounding FAult Locating}

\subsection{Signal Injection Method}

Signal injection equipment consists of disturbance load, fault detection equipment (FTU, DTU or fault indicator). The basic principle of the signal injection is injecting the signal current into the fault system during failure and realizing the fault locating by tracking and detecting the path and characteristics of injected signals. According to the different injection signal, the signal injection method is divided into small signal injection method and large signal injection method. The small signal injection method has the "S injection method" ${ }^{10]}$, and the large signal injection method has the variable frequency signal injection method ${ }^{[11]}$ and the remnant current increment method ${ }^{[12]}$. Next, each method is introduced in the following.

\subsubsection{S Injection Method}

The $S$ injection method belongs to small signal injection method. This method is realized by injecting a certain frequency of Ac current signal to the grounding line through the neutral point of the threephase voltage transformer after grounding fault in the distribution network. The injection signal can be formed through the circuit of fault line, fault locating, the ground and three-phase voltage transformer. By detecting each feeder using signal detector, we can judge that the line that flows through the injected signal is the fault line.

This method injects signal using the ground phase PT which is not working, and there is no additional equipment and no adverse effects on the running device. Besides, the detection will not be affected by any inherent signal. However, the injection signal is generally small because of influence of the capacity of the voltage transformer. In particular, the detection effect is not well when the ground resistance is large or the locating has intermittent arc.

\subsubsection{Variable Frequency Signal Injection Method}

The variable frequency signal injection method is large signal injection method. It can efficiently solve the question when the ground resistance is large. The principle is to select the arc suppression coil transformer or fault phase voltage transformer to inject variable frequency constant current signal, according fault displacement voltage and fault voltage. Then, monitor the change of the zero sequence current Angle and damping rate. Finally, obtain the locating criterion by comparing the damping ratio of each line and the other factors such as moisture and insulation aging.

When the grounding resistance is small, the signal current mostly flows through the fault line. This will cause a large damping rate on the non-fault line, and the failure accuracy will not be guaranteed.

\subsubsection{Remnant Current Increment Method}

The remnant current increment method also belongs to large signal injection method, and it is applicable to the resonant grounding system. The basic principle is that under the condition of singlephase grounding fault in power grid, if increase the mistuning degree of arc suppression coil (or change the resistance of the limiting resistance), the zero-sequence current of the fault line will increase. This method is established on the rapid processing and comprehensive analysis of microcomputer. The concrete process includes three steps. Firstly, collect the zero-sequence current of the outgoing lines after the single-phase grounding fault occurs. Then, change the compensation of the arc suppression coil and collect the zero-sequence current of the outgoing lines again. Finally, compare the change of zero-sequence current and select the largest changed one, and it is the fault line that we are looking for.

The remnant current increment method has advantages of simple principle, high sensitivity and reliability. And it is not affected by measurement errors produced by equipment such as current transformer. But, this method needs to add special equipments, and it is restricted by operation, management, site and other factors. In some cases it is not acceptable to the operation department.

\subsection{Fault Locating Method Based on Steady State Signals}

The signal injection method requires additional signal devices and the engineering implementation is complex. Whether it is a neutral grounding system, an arc suppression coil grounding system, or a neutral non-grounding system, there is a common feature that they all produce zero-sequence changes after the single-phase grounding fault occurs. By analyzing, we know that the zero-sequence current 
lags behind the zero-sequence voltage $90^{\circ}$ in the fault line. But the zero-sequence voltage lags behind the zero-sequence current $90^{\circ}$ in the non-fault line. In additional the zero-sequence current amplitude is the maximum in the fault line.

Therefore, the fault locating method based on steady state signals can be carried out. According to different study subjects, this method can be divided into zero-sequence current comparison of amplitude, zero-sequence current comparison of phase, method based on active power component of zero-sequence current and method based on negative-sequence current. Next, all methods will be introduced.

\subsubsection{Zero-Sequence Current Comparison of Amplitude}

When the neutral non-grounding system occur single-phase grounding fault, the zero sequence current of the fault line is bigger than the non-fault line and it is equal to the sum of the capacitance current of all non-fault elements to the ground. So we can locate the fault by comparing the zero-sequence current amplitude of each line.

This method can be misjudged when there is a long line in the system, and its locating capability will be greatly reduced in the arc suppression coil grounding system. Besides, this method can be affected by the imbalance of current transformer, length of line, operation mode of system and transition resistance, the sensitivity is low.

\subsubsection{Zero-Sequence Current Comparison of Phase}

When the single-phase grounding fault occurs, zero-sequence current in the fault line lags behind the zero-sequence voltage $90^{\circ}$. But in the non-fault line, the zero-sequence voltage lags behind the zerosequence current $90^{\circ}$. The direction of the zero-sequence current in the fault line is opposite of the direction of zero-sequence current in the non-fault line. It is the principle that the zero-sequence current comparison of phase realize.

Due to the "pointer effect", the phase judgment is difficult when the outgoing line is shorter and the zero-sequence current is small. In this case, this method is not applicable. In addition, because the influence of transition resistance and unbalanced current is great, it is also not applicable to the system operation mode of the arc suppression coil grounding system.

\subsubsection{Active Power Component of Zero-Sequence Current}

Because of grounding conductance and resistance losses of arc suppression coil, the fault current contains the active component when single-phase grounding fault occurs in the distribution network. And, the active component of the fault line is larger than the non-fault line and the direction is opposite. It is the feature that the active power component of zero-sequence current uses to form a protective criterion. In a concrete device, the zero-sequence active power of each line can be calculated using zero sequence voltage and zero sequence current. Then, we can locate the fault by comparing the direction of the zero-sequence active power of each line.

Although this method is not affected by the arc suppression coil, the active component of the fault current is very small and the imbalance of the three-phase parameters of the line will have an effect, the sensitivity is also low.

\subsubsection{Method Based on Negative-Sequence Current}

When the single-phase grounding fault occurs in the distribution network, the negative-sequence source impedance is relatively small. So the negative-sequence current flows mostly from the fault point to the generation, and the negative-sequence current of the non-fault lines is relatively small. The method based on negative-sequence current forms the protection criterion by using the negativesequence current distribution.

This method has strong resistance to transition resistance and has strong resistance to arc-light grounding. The disadvantages of this method are that there are large negative-sequence currents in the normal operation of the system, and the acquisition of negative-sequence current is not as simple and accurate as zero-sequence current.

\subsection{Fault Locating Method Based on Transient Signals}

Except signal injection and steady state signals, we can locate the fault by analyzing the transient signal. After the single-phase grounding fault, there is a transient process that lasts a number of cycles. 
In this process, the zero-sequence current of transient fault is several times or even dozens of times larger than the steady-state value and is not affected by the arc suppression coil. This method described by the principle is the fault locating method based on transient signals.

Under the premise of high reliability of information detection and transmission, this method has high locating accuracy and can handle all kinds of grounding faults and is not affected by the grounding mode. Therefore, it is the most widely used single-phase fault locating method. Depending on how the object is treated, this method can be divided into the first half wave method, the method based on transient feature frequency band and the method based on wavelet analysis. Next, all methods will be introduced briefly.

\subsubsection{First Half Wave Method}

The first half wave method is based on the assumption that the fault occurs near the maximum phase voltage. At this point, the fault phase capacitor discharges through the fault line to the fault point, making the short-circuit current of the fault line and the first half wave of the non-fault line in the opposite direction.

This method can only be applied to the fault grounding phase voltage near the peak, there may be misjudged when the fault grounding phase voltage near 0 . The time determining the first half-wave polarity relationship is short (far less than the transient process) and it is affected by the line structure and parameters, the detection reliability is low.

\subsubsection{Method Based on Transient Feature Frequency Band}

This method studies the phase-frequency characteristics of the zero-sequence impedance of the nonfault line and fault line. According to the phase-frequency characteristic, the frequency band is divided into different segments and the first capacitive characteristic frequency band is defined. In this frequency band, the zero-sequence current amplitude of the fault line is the largest, and the direction of the zero-sequence current of the non-fault line is opposite to the direction of the zero sequence current of the fault line. This method makes use of this characteristic of the transient zero-sequence current in this band to form a protective criterion.

This method is not affected by the arc suppression coil. But in the fault process (especially in the intermittent arc grounding), the difference between the direction parameters of fault lines and nonfault lines is not always present (it could be 0 at the same time).

\subsubsection{Method Based on Wavelet Analysis}

Wavelet analysis is a signal processing method. Because of its advantages in signal decomposition, reconstruction, feature extraction and signal-noise separation, wavelet analysis has a broad application prospect in power system. The basic principle of fault locating technique based on wavelet analysis is to use wavelet function which time and frequency are finite to replace the steady-state sinusoidal signal as the basis function to decompose the transient signal. It can better reflect the characteristics of the frequency component of the transient signal with time change, especially for the transient mutation signal and small signal. According to the amplitude of the transient zero-sequence current of the fault line is higher than the non-fault line and they go in the opposite direction, the method uses the suitable wavelet transform to transform the instantaneous current signal and extract the subcomponent and select the fault line. The technical difficulty of wavelet analysis is that the selection of wavelet basis function and wavelet decomposition scale lacks theoretical basis.

\section{CONCLUSION AND PROSPECT}

The research on single-phase grounding fault locating has achieved a lot of achievements over the years. This paper reviews mainstream locating technologies. However, due to the complexity of the distribution system itself, the equipment based on existing technology cannot adapt to the operating conditions of various systems. In order to realize the accurate and fast isolation of single-phase grounding fault and improve the reliability of power supply, new fault locating technology applying power distribution automation information and power quality monitoring information should be studied to realize fault locating.

\section{ACKNOWLEDGEMENT}

The authors would like to acknowledge gratefully the support of Qionghai Power Supply Bureau, China for Research and Application of Distribution Network Fault Location Technology Based on Limited Measurements (070400kk52170001). 


\section{REFERENCES}

[1] WANG Yong. Study of Power System Operational Reliability Analysis and Evaluation [D]. Shandong University, 2012.

[2] CHEN GuangXin. Research for Fault Recognition of Distribution Line Basing on Clustering Artificial Immune Network [D]. East China Jiaotong University, 2014.

[3] XUE Yong-duan, XU Bing-yin, FENG Zu-ren, edal. The Principle of Directional Earth Fault Protection Using Zero Sequence Transients in Non-solid Earthed Network [J]. Proceedings of the CSEE, 2003, (07): 51-56.

[4] Zhu J, Lubkeman DL, Girgis AA. Automated fault locating and diagnosis on electric power distribution feeders [J]. IEEE Transactions on Power Delivery, 1997, 12(2): 801-809.

[5] Mu Longhua, Meng Qinghai. Ground-fault Protection Based on Active Component of Zero-Sequence Current in Resistance Neutral Grounded Distribution Systems [J]. Proceedings of IEEE TENCON, 2002, (2): 1897-1990.

[6] ZENG Xiangjun, YIN Xianggen, ZHANG Zhe, edal. Study for Negative Sequence Current Distribution and Ground Fault Protection in Middle Voltage Power Systems [J]. Proceedings of the CSEE, 2001, (06): 85-90.

[7] XIAO Bai, SHU Hongchun, GAO Feng. A Survey on Faulty Line Selection Technology for Single-phase Grounded Transmission Line in Small Current Neutral Grounded System [J]. Relay, 2001, (04): 16-20.

[8] Pourahmadi-nakhli M, Safavi AA. Path characteristic frequency-based fault locating in radial distribution systems using wavelets and neural networks [J]. IEEE Transactions on Power Delivery, 2011, 26(2): 772 781.

[9] Borghetti A, Bosetti M, Nucci CA, et al. Integrated use of time-frequency wavelet decompositions for fault locating in distribution networks: theory and experimental validation [J]. IEEE Transactions on Power Delivery, 2010, 25(4): 3139-3146.

[10] Chen Chen, Li Linkai, Zhang Qiao. Online Inductor Parameters Identification by Small-Signal Injection for Sensorless Predictive Current Controlled Boost Converter [J]. IEEE Transactions on Industrial Informatics, 2013, (13): 1554-1564.

[11] ZENG Xiangjun, YIN Xianggen, YU Yongyuan, edal. New Methods for Control and Protection Relay in a Compensated Medium Voltage Distribution Network Based on Injecting Various Frequency Current [J]. Proceedings of the CSEE, 2000, (01):30-33+37.

[12] WANG Yumei, GUO Hao, HUI Shengda. Fault Locating Method of Resonant Grounding System Based on Residual Current Incremental Method [J]. Automation of Electric Power System, 2011, (02): 62-65+92.

\section{AUTHORS' BIOGRAPHY}

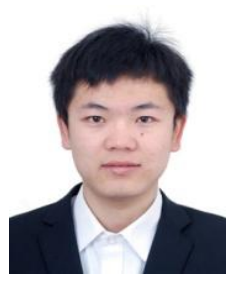

Yanqi Zhang, received the B.S.E.E. degree from Wuhan University in 2016. Now he is pursing the master degree in school of electrical engineering at Wuhan University. His research field is power system operation.

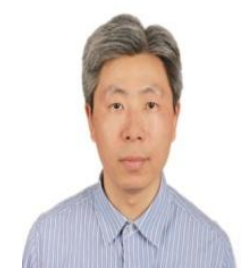

Tao Lin, is a professor of school of electrical engineering at Wuhan University. His research fields are power system relaying, power quality, power system operation and control.

Citation: Tao Lin et al. (2017). Review of Single-Phase Grounding Fault Locating in Distribution Network, International Journal of Research Studies in Electrical and Electronics Engineering (IJRSEEE), 3(3), pp.1-5, DOI: http://dx.doi.org/10.20431/2454-9436.0303001.

Copyright: (C) 2017 Tao Lin. This is an open-access article distributed under the terms of the Creative Commons Attribution License, which permits unrestricted use, distribution, and reproduction in any medium, provided the original author and source are credited 